\title{
Correlation of Serum Cytokines in Patients with Post-Traumatic Stress Disorder
}

\author{
Peixue Chang1, Min Guo ${ }^{2}$ (1) \\ ${ }^{1}$ Department of Endocrinology, The Fourth People's Hospital of Haikou, Haikou, China \\ ${ }^{2}$ Department of Psychology, Hainan General Hospital, Haikou, China \\ Email: 171582081@qq.com
}

How to cite this paper: Chang, P.X. and Guo, M. (2021) Correlation of Serum Cytokines in Patients with Post-Traumatic Stress Disorder. Journal of Behavioral and Brain Science, 11, 281-285.

https://doi.org/10.4236/jbbs.2021.1112023

Received: October 31, 2021

Accepted: December 14, 2021

Published: December 17, 2021

Copyright () 2021 by author(s) and Scientific Research Publishing Inc. This work is licensed under the Creative Commons Attribution International License (CC BY 4.0).

http://creativecommons.org/licenses/by/4.0/

\section{(c) (i) Open Access}

\begin{abstract}
Objective: The objective is to investigate the correlation between post-traumatic stress disorder (PTSD) and serum cytokines in Hainan. Methods: The serum levels of IL-2, IL-4, IL- 6 and IL-8 were detected by enzyme-linked immunosorbent assay (ELISA) in 60 post-traumatic stress disorder patients and 60 healthy controls in Hainan. Results: The serum levels of IL-2 $(t=15.981, P=$ $0.000)$, IL-6 $(\mathrm{t}=20.984, \mathrm{P}=0.000)$ and IL-8 $(\mathrm{t}=6.906, \mathrm{P}=0.000)$ in PTSD group were significantly higher than those in control group. There was no significant difference in serum IL-4 content between the two groups $(t=$ $1.109), P=0.259)$. Conclusion: POST-traumatic stress disorder is closely related to the changes of serum cytokines, and IL-2, IL- 6 and IL- 8 may play an important role in the pathogenesis of PTSD.
\end{abstract}

\section{Keywords}

Post-Traumatic Stress Disorder, Cytokines, IL-2, IL-4, IL-6, IL-8

\section{Introduction}

Post-traumatic stress disorder (PTSD) is a typical psychological stress disorder. Post-traumatic stress disorder (PTSD) is a kind of post-traumatic psychological imbalance, which is a delayed and/or lasting anxiety response to unusually threatening and catastrophic events [1]. Recent studies have shown that post-traumatic stress disorder is a disease state caused by the joint involvement of the neuroendocrine system, central nervous system and immune system. In addition to major natural disasters, general man-made disasters are also very common in China. According to China's injury prevention report, injuries of various types occur about 200 million times in China every year, resulting in about 700,000 750,000 deaths, an average of 2000 people a day. Studies have shown that $50 \%$ - 
$70 \%$ of individuals are exposed to traumatic events. Normally, the incidence of PTSD in the general population is $0.2 \%$. Due to its special geographical location and geological structure, Hainan province is an island province prone to different types of natural disasters such as typhoon, flood, earthquake, tsunami and fire. This study compared the levels of serum interleukin between PTSD patients and the control group in Hainan area, in order to preliminarily explore the correlation between serum cytokines and the incidence of PTSD, and provide a scientific basis for the clinical diagnosis of PTSD patients.

\section{Objects and Methods}

\subsection{Subjects}

All the cases were from Han nationality patients diagnosed with PTSD who sought help in the psychological outpatient department of Haikou Fourth People's Hospital and Hainan Provincial People's Hospital from January 2019 to December 2019.

Inclusion criteria: 1) Age was 35-65 years old; 2) It meets the diagnostic criteria of PTSD in DSM-4. Exclusion criteria; Associated with mental retardation, personality disorders, substance or alcohol dependence, pregnancy and major diseases. There were 29 males and 28 females in the study group with an average age of $43.20 \pm 7.03$ years. There were 31 males and 26 females in the control group, with an average age of $45.02 \pm 7.07$ years, and the inclusion criteria were as follows: there was no PTSD disease, the rest were matched with PTSD group. All subjects gave informed consent to the experiment and signed informed consent, which was approved by the hospital's clinical experiment Ethics Committee.

\subsection{Methods}

$2 \mathrm{ml}$ of venous blood from the middle of elbow was taken from all subjects on an empty stomach in the morning. Samples were taken from PTSD patients within 1 week after diagnosis, and the content of cytokines was determined by enzyme-linked immunosorbent assay (ELISA), which was produced by Shanghai Jianglai Biotechnology Co., LTD.

Statistical Analysis SPSS 13.0 statistical software was used for statistical analysis. The measurement data were expressed as mean \pm standard deviation (). T-test was used for comparison of mean between the two groups, logistic regression analysis was used for multivariate analysis, and the test level was 0.05.

\section{The Results}

1) Comparison of basic conditions between the study group and the control group

a) There was no statistically significant difference in gender composition between the study group (PTSD) and the control group $(\mathrm{P}>0.05)$, as shown in Table 1.

b) There was no statistically significant difference in age between the study 
group (PTSD) and the control group $(\mathrm{P}>0.05)$, as shown in Table 2.

2) Comparison of serum cytokines contents between the study group (PTSD) and the control group

The serum levels of IL-2, IL-6 and IL-8 in the PTSD group from Hainan area were significantly higher than those in the control group, with statistical significance $(\mathrm{P}<0.01)$. There was no significant difference in serum IL-4 content between the two groups $(t=1.109, P=0.259)$. See Table 3 .

\section{Discussion}

Cytokines are divided into two categories: proinflammatory factors (e.g., IL-2, IL-6, IL-8, TNF) and anti-inflammatory factors (e.g., IL-4, IL-10, IL-13). The role of proinflammatory factors is to stimulate immune cells and exert local and remote effects on damaged cells [2]. The results of this study showed that the serum levels of proinflammatory cytokines il-2 and il-6 in PTSD patients were significantly different from those in the non-PTSD group, and higher than those in the control group. Some foreign research data [3] [4] showed that the plasma levels of cytokines il-2 and il-6 increased in PTSD patients. Moreover, plasma IL-2 and IL-6 levels were positively correlated with the severity of depressive symptoms and HPA axis activity, which could be used as a test indicator of PTSD. In addition, [5] [6] [7] showed that the expressions of IL-1, IL-6 and TNF- $\alpha$ were increased in PTSD patients, suggesting that PTSD is related to the activation of inflammatory response immune system and is a disorder of neuro-psychological neuro-psychological immune system. However, it was also reported that the

Table 1. Comparison of gender composition between the PTSD group and the control group.

\begin{tabular}{ccccc}
\hline group & male & female & $\chi^{2}$ & P \\
\hline PTSD group & 29 & 28 & 0.143 & 0.797 \\
Control group & 31 & 26 & &
\end{tabular}

Table 2. Comparison of age between the PTSD group and the control group.

\begin{tabular}{ccccc}
\hline group & N & Age (years) & t & P \\
\hline PTSD group & 57 & $43.20 \pm 7.03$ & 1.497 & 0.140 \\
Control group & 57 & $45.02 \pm 7.07$ & & \\
\hline
\end{tabular}

Table 3. Comparison of age between the PTSD group and the control group (PG/ml).

\begin{tabular}{cccccc}
\hline group & N & IL-2 & IL-4 & IL-6 & IL-8 \\
\hline PTSD group & 57 & $19.01 \pm 5.04$ & $7.03 \pm 2.29$ & $23.29 \pm 5.01$ & $54.03 \pm 18.99$ \\
Control group & 57 & $7.20 \pm 2.51$ & $7.40 \pm 1.99$ & $6.98 \pm 3.19$ & $32.06 \pm 13.50$ \\
t & & 15.981 & 1.109 & 20.984 & 6.906 \\
P & 0.000 & 0.259 & 0.000 & 0.000 \\
\hline
\end{tabular}


LEVEL of IL-2 in PTSD group was significantly lower than that in normal group. However, [8] showed no significant difference in IL-2, IL-6 and IL-10. The levels of IL-2, IL-6, IL- 8 and TNF- $\alpha$ in PTSD patients were higher than those in control group. These results suggest that oxidative stress, inflammatory response and adrenergic system may play an important role in the pathogenesis of PTSD [9]. The results of this study are inconsistent with the conclusions of domestic and foreign scholars, mainly because different patients were recruited in this study, and there are differences in the different time course and severity of PTSD. The results also showed higher levels of IL-8 than the non-PTSD group. Studies have shown [6] that IL-8 can promote leukocyte infiltration in brain parenchyma, and then leukocyte protease activation and free radical formation can lead to lipid peroxidation and neuron damage, and anti-IL- 8 antibody can significantly reduce the volume of cerebral infarction and the degree of cerebral edema, and relieve patients' pain [10]. This is consistent with the results of imaging suggesting that PTSD patients may be accompanied by brain damage (atrophy of hippocampus and other brain areas, etc.) [5]. IL-4, as Th2 cell autoactivating factor, can promote B cell proliferation and differentiation as well as acetylcholine receptor antibody and is considered to be one of the main factors mediating humoral immune response [11]. The results of this study showed that there was no significant difference in serum IL-4 content.

\section{Conclusion}

Therefore, the levels of IL-2, IL-4 and IL-8 were higher than those of the control group, suggesting that cytokines and proinflammatory factors may play an important role in stimulating the immune system during the pathogenesis of PTSD, which also provides a new idea for further study on the pathogenesis of PTSD.

\section{Conflicts of Interest}

The authors declare no conflicts of interest regarding the publication of this paper.

\section{References}

[1] Kim, G.S., Smith, A.K., Xue, F., Michopoulos, V., Adriana, L., et al. (2019) Methylomic Profiles Reveal Sex-Specific Differences in Leukocyte Composition Associated with Post-Traumatic Stress Disorder. Brain Behavior and Immunity, 81, 280-291. https://doi.org/10.1016/j.bbi.2019.06.025

[2] Guo, J.-C., Tian, Z.-L., Wang, X.-D., Guo, M., et al. (2016) Post-Traumatic Stress Disorder after Typhoon Disaster and Its Correlation with Platelet 5-HT Concentrations. Asian Pacific Journal of Tropical Medicine, 9, 913-915. https://doi.org/10.1016/j.apjtm.2016.07.011

[3] Guo, J.-C., Guo, M., et al. (2018) Correlations of Four Genetic Single Nucleotide Polymorphisms in Brain-Derived Neurotrophic Factor with Posttraumatic Stress Disorder. Psychiatry Investigation, 15, 407-412. https://doi.org/10.30773/pi.2017.06.17.1

[4] Guo, M., Cheng, T., Guo, J.-C., et al. (2012) Study on Serum Cytokine Levels in 
Posttraumatic Stress Disorder Patients. Asian Pacific Journal of Tropical Medicine, 5, 323-325. https://doi.org/10.1016/S1995-7645(12)60048-0

[5] Guo, J.-C., Yang, Y.-J., Zheng, J.-F., et al. (2019) Functional rs6265 Polymorphism in the BDNF Gene Confers protection against Neurocognitive Dysfunction in Posttraumatic Stress Disorder among Chinese Patients with Hepatocellular Carcinoma. Journal of Cellular Biochemistry, 120, 10434-10443.

https://doi.org/10.1002/jcb.28328

[6] Lang, X.Q., Lin, L., Ji, L., Yuan, X.Y., et al. (2017) P2X3 Receptor-Mediated Visceral Hyperalgesia and Neuronal Sensitization Following Exposure to PTSD-Like Stress in the Dorsal Root Ganglia of Rats. Neurogastroenterology \& Motility, 29, e12976. https://doi.org/10.1111/nmo.12976

[7] Guo, M., Chen, F., Guo, J.-C., Jiang, X.-L., et al. (2012) Study of the Hippocampus and the Anterior Cingulated Gyrus by Proton MR Spectroscopy in Patients with Post-Traumatic Stress Disorder. Asian Pacific Journal of Tropical Medicine, 5, 162-164. https://doi.org/10.1016/S1995-7645(12)60017-0

[8] George, S.A., Stout, S.A., Tan, M., Dayan, K., et al. (2013) Early Handling Attenuates Enhancement of Glucocorticoid Receptors in the Prefrontal Cortex in an Animal Model of Post-Traumatic Stress Disorder. Biology of Mood \& Anxiety Disorders, 3, Article No. 22. https://doi.org/10.1186/2045-5380-3-22

[9] Zhou, S.P., et al. (2013) Analysis of Cytokines and Cortisol Levels in Patients with Post-Traumatic Stress Disorder. Chinese Journal of Tropical Medicine, 13, 922-924

[10] Guo, J.-C., Jiang, X.-L., Yang, Y.-J., Zheng, X.-A., et al. (2018) CpG Methylation of Brain-Derived the Neurotrophic Factor Gene Promoter as a Potent Diagnostic and Prognostic Biomarker for Post-Traumatic Stress Disorder. International Journal of Clinical and Experimental Pathology, 11, 5101-5109.

[11] Hao, J.M. and Zhang, Z.G. (2018) Research Progress of Proteomics of Chinese Herbal Compound Improving Post-Traumatic Stress Disorder. Chinese Journal of Veterinary Medicine, 37, 37-39. 